\title{
Model-Independent Results for SU(3) Violation in Twist-3 Light-Cone Distribution Functions
}

\author{
Jiunn-Wei Chen, Hung-Ming Tsai and Ke-Chuan Weng \\ Department of Physics, National Taiwan University, Taipei, Taiwan 10617
}

\begin{abstract}
Using chiral symmetry we investigate the leading $\mathrm{SU}(3)$ violation in the complete set of quark twist-3 light-cone distribution functions of the pion, kaon, and eta, including the two-parton distributions $\phi_{M}^{p}, \phi_{M}^{\sigma}$, and the three-parton distribution $\phi_{M}^{3}$. It is shown that terms non-analytic in the quark masses do not affect the shape, and only appear in the normalization constants. Predictive power is retained including the leading analytic $m_{q}$ operators. With the symmetry violating corrections we derive useful model-independent relations between $\phi_{\pi}^{p, \sigma, 3}, \phi_{\eta}^{p, \sigma, 3}, \phi_{K^{+}, K^{0}}^{p, \sigma, 3}$, and $\phi_{\bar{K}^{0}, K^{-}}^{p, \sigma}$. We also comment on the calculations of the moments of these distributions using lattice QCD and light-cone sum rules.
\end{abstract}




\section{INTRODUCTION}

Meson light cone distribution functions (LCDFs) play important roles in high energy hadronic exclusive processes [1-5]. The same LCDFs contribute in many processes relevant to measuring fundamental parameters of the Standard Model [6], such as $B \rightarrow \pi \ell \nu, \eta \ell \nu$ which give the Cabibbo-Kobayashi-Maskawa (CKM) quark-mixing matrix element $\left|V_{u b}\right|, B \rightarrow D \pi$ used for tagging, and $B \rightarrow \pi \pi, K \pi, K \bar{K}, \pi \eta, \ldots$ which are important for measuring CP violation.

With the increasing accuracy in data from the $B$ factories, the flavor dependence in LCDFs becomes important to understand the flavor symmetry breaking in processes like $B \rightarrow M M^{\prime}$ and $B \rightarrow M V$, where $M$ and $V$ are pseudoscalar and vector mesons, respectively. In Ref. [7], chiral perturbation theory (ChPT) was first applied to study the leading SU(3) symmetry breaking effects in twist-2 LCDFs $\phi_{M}^{P}(x)$. It was shown that terms non-analytic in the quark masses do not affect the shape, and only appear in the normalization constants. Furthermore, with the symmetry violating corrections useful model-independent relations between $\phi_{\pi}^{P}, \phi_{\eta}^{P}, \phi_{K^{+}, K^{0}}^{P}$, and $\phi_{\bar{K}^{0}, K^{-}}^{P}$ were derived.

Recently ChPT has also been applied to the computation of hadronic twist-2 matrix elements $[8,9]$. Many applications have been worked out, e.g., chiral extrapolations of lattice data [10-13], generalized parton distributions [14-16], large $N_{C}$ relations among nucleon and $\Delta$-resonance distributions [9], soft pion productions in deeply virtual Compton scattering [1719], pion-photon transition distributions [20] and exclusive semileptonic B decays [21]. The method is also generalized to the multi-nucleon case [19, 22].

In this paper, we apply ChPT to higher twist matrix elements. We study the leading $\mathrm{SU}(3)$ symmetry breaking in the complete set of quark twist-3 LCDFs, including the twoparton distributions $\phi_{M}^{p}, \phi_{M}^{\sigma}$, and the three-parton distribution $\phi_{M}^{3}$. Although those twist3 contributions are parametrically suppressed by inverse powers of large scales, they are numerically important in $B \rightarrow M M^{\prime}, B \rightarrow M V$ [2] and the meson electromagnetic form factor [23].

In the following sections, we will first summarize our main results on the leading $\mathrm{SU}(3)$ symmetry breaking of LCDFs, then study the three twist-3 LCDFs sequentially. Finally, we

will comment on calculations of those quantities using lattice QCD [24-26] and light cone sum rules $[27-32]$. 


\section{SUMMARY OF RESULTS}

The two-parton LCDFs for pseudoscalar meson $M$ are defined by the matrix element of the quark bilinear operator [34]

$$
\begin{aligned}
\left\langle M^{b}(p)\left|\bar{q}_{\beta}^{\prime}\left(\frac{y}{2} n\right)\left[\frac{y}{2} n,-\frac{y}{2} n\right] \lambda^{a} q_{\alpha}\left(-\frac{y}{2} n\right)\right| 0\right\rangle= & \frac{i}{4} \delta^{a b} \int_{0}^{1} d x e^{i(x-1 / 2) y p \cdot n}\left\{f_{M}^{P} \not \gamma_{5} \phi_{M}^{P}(x, \mu)\right. \\
& \left.-\gamma_{5}\left(f_{M}^{p} \phi_{M}^{p}(x, \mu)-\frac{y}{6} f_{M}^{\sigma} \sigma_{\mu \nu} p^{\mu} n^{\nu} \phi_{M}^{\sigma}(x, \mu)\right)\right\}_{\alpha \beta}
\end{aligned}
$$

where $n$ is a constant light-like vector, $n^{2}=0$ and our octet matrices are normalized so that $\operatorname{tr}\left[\lambda^{a} \lambda^{b}\right]=\delta^{a b} \cdot\left[\frac{y}{2} n,-\frac{y}{2} n\right]$ denotes the Wilson line connecting the quark bilinear located at different space-time points on a light cone. $\phi_{M}^{P}$ is a twist-2 LCDF while $\phi_{M}^{p}$ and $\phi_{M}^{\sigma}$ are twist-3 LCDFs for pseudoscalar meson $M . x$ is the quark momentum fraction and $\mu$ is the perturbative QCD renormalization scale. For simplicity we work in the isospin limit and the $\overline{\mathrm{MS}}$ scheme, and normalize the distributions so that $\int d x \phi_{M}^{i}(x, \mu)=1$ with $i=P, p, \sigma$.

Generically from chiral symmetry the leading order SU(3) violation for $\phi_{M}^{i}$ takes the form $[M=\pi, K, \eta]$

$$
\begin{aligned}
\phi_{M}^{i}(x, \mu)= & \phi_{0}^{i}(x, \mu)+\sum_{N=\pi, K, \eta} \frac{m_{N}^{2}}{(4 \pi f)^{2}}\left[E_{M}^{N, i}(x, \mu) \ln \left(\frac{m_{N}^{2}}{\mu_{\chi}^{2}}\right)\right. \\
& \left.+F_{M}^{N, i}\left(x, \mu, \mu_{\chi}\right)\right] .
\end{aligned}
$$

The functions $\phi_{0}^{i}, E_{M}^{N, i}$, and $F_{M}^{N, i}$ are independent of $m_{q}$, and are only functions of $\Lambda_{\mathrm{QCD}}$, $\mu$, and $x . F_{M}^{N, i}$ also depends on the ChPT dimensional regularization parameter $\mu_{\chi}$ which cancels the $\ln \left(m_{N}^{2} / \mu_{\chi}^{2}\right)$ dependence, so by construction $\phi_{M}^{i}$ is $\mu_{\chi}$ independent. Throughout the text, $\eta$ denotes the purely octet meson.

Using ChPT, we found very similar results to the twist-2 case [7] at leading order in $S U(3)$ violation $\left[\mathcal{O}\left(m_{q}\right)\right]$ :

1) The twist-2 and twist-3 LCDFs are analytic in $m_{q}$, meaning that

$$
E_{M}^{\pi, i}(x)=0, \quad E_{M}^{K, i}(x)=0, \quad E_{M}^{\eta, i}(x)=0
$$

The leading logarithmic corrections can all be absorbed by the normalization constants $f_{M}^{i}$.

2) By charge conjugation and isospin symmetry,

$$
\phi_{\pi}^{i}(x)=\phi_{\pi}^{i}(1-x), \quad \phi_{\eta}^{i}(x)=\phi_{\eta}^{i}(1-x)
$$


And by isospin symmetry

$$
\phi_{K^{+}}^{i}(x)=\phi_{K^{0}}^{i}(x), \quad \phi_{K^{-}}^{i}(x)=\phi_{\bar{K}^{0}}^{i}(x)
$$

These two equations are true to all orders in $m_{q}$.

3)

$$
\phi_{K^{+}}^{i}(x)-\phi_{K^{+}}^{i}(1-x)=\phi_{K^{-}}^{i}(1-x)-\phi_{K^{-}}^{i}(x)=\left(m_{s}-\bar{m}\right) \delta \phi^{i}(x),
$$

where $\delta \phi^{i}(x)$ is $m_{q}$ independent.

4) Gell-Mann-Okubo-like relations exist among the octet mesons

$$
\phi_{\pi}^{i}(x)+3 \phi_{\eta}^{i}(x)=2\left[\phi_{K^{+}}^{i}(x)+\phi_{K^{-}}^{i}(x)\right] .
$$

5) The three-parton LCDFs also have relations similar to 1)-4) [see Eqs.(66-68)].

6) Statements 1)-5) are still true in quenched and partially quenched simulations, and with the leading finite volume and finite lattice spacing corrections.

7) The light cone sum rule results [32] for twist-3 Gegenbauer moments are consistent with the ChPT prediction $[i=p, \sigma]$

$$
4 a_{2}^{K, i}=a_{2}^{\pi, i}+3 a_{2}^{\eta, i}
$$

The analogous ChPT relation for twist-2 moments puts a tight constraint on the numerical values of $a_{2}^{M, P}$.

\section{TWO-PARTON LCDFS}

The operator product expansion of the non-local quark bilinear operator in Eq.(1) gives rise to the twist-2 operator $O_{k}^{P, a}$ and twist-3 operators $O_{k}^{p, a}$ and $\left(O_{k}^{\sigma, a}\right)^{\mu \nu}$ :

$$
\begin{aligned}
O_{k}^{P, a} & =\bar{\psi} \not h \gamma_{5} \lambda^{a}(i n \cdot \overleftrightarrow{D})^{k} \psi \\
O_{k}^{p, a} & =\bar{\psi} \gamma_{5} \lambda^{a}(i n \cdot \overleftrightarrow{D})^{k} \psi \\
\left(O_{k}^{\sigma, a}\right)^{\mu \nu} & =\bar{\psi} \sigma^{\mu \nu} \gamma_{5} \lambda^{a}(i n \cdot \overleftrightarrow{D})^{k+1} \psi
\end{aligned}
$$

where $\overleftrightarrow{D}=\overleftarrow{D}-\vec{D}$ and $k=0,1,2, \ldots$ Here having the vector indices dotted into $n^{\mu_{0}} \cdots n^{\mu_{k(+1)}}$ has automatically projected onto the symmetric and traceless part. The matrix 
elements of these operators yield

$$
\begin{aligned}
\left\langle M^{b}(p)\left|O_{k}^{P, a}\right| 0\right\rangle & =-i f_{M}^{P} \delta^{a b}(n \cdot p)^{k+1}\left\langle z^{k}\right\rangle_{M}^{P} \\
\left\langle M^{b}(p)\left|O_{k}^{p, a}\right| 0\right\rangle & =-i f_{M}^{p} \delta^{a b}(n \cdot p)^{k}\left\langle z^{k}\right\rangle_{M}^{p} \\
\left\langle M^{b}(p)\left|\left(O_{k}^{\sigma, a}\right)^{\mu \nu}\right| 0\right\rangle & =-\frac{k+1}{3} f_{M}^{\sigma} \delta^{a b}(n \cdot p)^{k}\left(p^{\mu} n^{\nu}-p^{\nu} n^{\mu}\right)\left\langle z^{k}\right\rangle_{M}^{\sigma},
\end{aligned}
$$

where $z \equiv 1-2 x$ and the moments are defined as

$$
\left\langle z^{k}\right\rangle_{M}^{i}=\int_{0}^{1} d x(1-2 x)^{k} \phi_{M}^{i}(x) .
$$

Following similar procedures as in the twist-2 case [7], we analyze the twist-3 matrix elements.

\section{A. The $\phi_{M}^{p}$ Analysis}

It is convenient to write

$$
O_{k}^{p, a}=O_{k, L R}^{p, a}-O_{k, R L}^{p, a}
$$

where

$$
O_{k, L R}^{p, a}=\bar{\psi}_{L} \lambda_{L R}^{a}[i n \cdot \overleftrightarrow{D}]^{k} \psi_{R}
$$

and similarly for $O_{k, R L}^{p, a} . \quad \psi_{L, R}=\left[\left(1 \mp \gamma_{5}\right) / 2\right] \psi$ is the left(right)-handed quark field. The distinction between $\lambda_{L R}^{a}$ and $\lambda_{R L}^{a}$ is only for bookkeeping purposes. We will set $\lambda_{L R}^{a}=$ $\lambda_{R L}^{a}=\lambda^{a}$ at the end.

When $a=3$ or $8, O_{k}^{p, a}$ transforms simply under charge conjugation $(\mathcal{C})$, being even when $k$ is even, and odd when $k$ is odd. The meson states $\pi^{0}$ and $\eta$ (i.e., $M^{3,8}$ ) are $\mathcal{C}$ even. Thus from Eq. (13), $\left\langle z^{k}\right\rangle_{\pi^{0}, \eta}^{p}$ vanishes when $k$ is odd due to $\mathcal{C}$ (and using isospin the same applies for $\left.M=\pi^{ \pm}\right)$. For all $a$ 's the operator would transform as

$$
\mathcal{C}^{-1} O_{k}^{p, a} \mathcal{C}=(-1)^{k} O_{k}^{p, a}
$$

if we demanded that, under the $\mathcal{C}$ transformation

$$
\lambda_{L R}^{a} \rightarrow \lambda_{L R}^{a T}, \quad \lambda_{R L}^{a} \rightarrow \lambda_{R L}^{a T}
$$

To construct the corresponding hadronic ChPT operators, we define $\Sigma=\exp \left(2 i \pi^{a} \lambda^{a} / f\right)$ and $m_{q}=\operatorname{diag}\left(\bar{m}, \bar{m}, m_{S}\right)=m_{q}^{\dagger}$. Under a chiral $S U(3)_{L} \times S U(3)_{R}$ transformation, we have

$$
\begin{aligned}
\Sigma \rightarrow L \Sigma R^{\dagger}, & m_{q} \rightarrow L m_{q} R^{\dagger} \\
\lambda_{L R}^{a} \rightarrow L \lambda_{L R}^{a} R^{\dagger}, & \lambda_{R L}^{a} \rightarrow R \lambda_{R L}^{a} L^{\dagger} .
\end{aligned}
$$


Under charge conjugation, $\Sigma \rightarrow \Sigma^{T}, m_{q} \rightarrow m_{q}^{T}$, while $\lambda_{L R, R L}^{a}$ transform according to Eq. (19). At next to leading order (NLO) in the $p^{2} / \Lambda_{\chi}^{2}$ and $m_{M}^{2} / \Lambda_{\chi}^{2}$ chiral expansion,

$$
O_{k}^{p, a} \rightarrow \sum_{i} c_{k, i} \mathcal{O}_{k, i}^{p, a}+\sum_{i} b_{k, i} \overline{\mathcal{O}}_{k, i}^{p, a}
$$

where the $\mathcal{O}$ 's are the leading order (LO) and the $\overline{\mathcal{O}}$ 's are NLO. The sum on $i$ runs over hadronic operators which have the same transformation properties as $O_{k}^{p, a}$. The ChPT Wilson coefficients $c_{k, i}$ and $b_{k, i}$ encode physics at the scale $p^{2} \sim \Lambda_{\chi}^{2}$ and the operators encode $p^{2} \ll \Lambda_{\chi}^{2}$.

At LO in the chiral expansion only one operator contributes in our analysis:

$$
\mathcal{O}_{k, 0}^{p, a}=\frac{f^{p} f}{8}\left[1+(-1)^{k}\right] \operatorname{Tr}\left[\lambda_{L R}^{a} \square^{k} \Sigma^{\dagger}-\lambda_{R L}^{a} \square^{k} \Sigma\right],
$$

where $\square^{k}=(\text { in } \cdot \partial)^{k}, f^{p}=f_{M}^{p}$ in the chiral limit. $\mathcal{O}_{k, 0}^{p, a}$ vanishes when $k$ is odd due to charge conjugation and $\mathrm{SU}(3)$ symmetry. The normalization of $\mathcal{O}_{k, 0}^{p, a}$ is chosen such that $c_{0,0}=1$.

Note that the operator

$$
\mathcal{O}_{k, 1}^{p, a}=\frac{f^{p} f}{8}\left[1+(-1)^{k}\right] \operatorname{Tr}\left[\lambda_{L R}^{a} \Sigma^{\dagger}\left(\square^{k} \Sigma\right) \Sigma^{\dagger}-\lambda_{R L}^{a} \Sigma\left(\square^{k} \Sigma^{\dagger}\right) \Sigma\right]
$$

is also LO but not independent of $\mathcal{O}_{k, 0}^{p, a}$. Since $\square^{k}\left(\Sigma^{\dagger} \Sigma\right)=0$,

$$
0=\left(\square^{k} \Sigma^{\dagger}\right) \Sigma+\Sigma^{\dagger}\left(\square^{k} \Sigma\right)+\ldots,
$$

where the ellipse denotes $\left(\square^{k-m} \Sigma^{\dagger}\right)\left(\square^{m} \Sigma\right)$ terms that only contribute for matrix elements with more than one meson. Thus, Eq. (25) allows us to move factors of $\square^{k}$ onto a neighboring $\Sigma$ and reduce $\mathcal{O}_{k, 1}^{p, a}$ to $\mathcal{O}_{k, 0}^{p, a}$.

At NLO there are two independent operators:

$$
\begin{aligned}
\overline{\mathcal{O}}_{k, 1}^{p, a}= & \frac{f^{p} f}{8}\left[1+(-1)^{k}\right] \operatorname{Tr}\left[m_{q} \Sigma^{\dagger}+\Sigma m_{q}^{\dagger}\right] \operatorname{Tr}\left[\lambda_{L R}^{a} \square^{k} \Sigma^{\dagger}-\lambda_{R L}^{a} \square^{k} \Sigma\right], \\
\overline{\mathcal{O}}_{k, 2}^{p, a}= & \frac{f^{p} f}{8} \operatorname{Tr}\left[\lambda_{L R}^{a}\left\{m_{q}^{\dagger} \Sigma \square^{k} \Sigma^{\dagger}+(-1)^{k}\left(\square^{k} \Sigma^{\dagger}\right) \Sigma m_{q}^{\dagger}\right\}\right. \\
& \left.-\lambda_{R L}^{a}\left\{m_{q} \Sigma^{\dagger} \square^{k} \Sigma+(-1)^{k}\left(\square^{k} \Sigma\right) \Sigma^{\dagger} m_{q}\right\}\right] .
\end{aligned}
$$

All other NLO operators have derivatives on more than one $\Sigma$, or can be reduced to $\overline{\mathcal{O}}_{k, 1}^{p, a}$ and $\overline{\mathcal{O}}_{k, 2}^{p, a}$ using the equations of motion. For instance, consider

$$
\begin{aligned}
\overline{\mathcal{O}}_{k, 3}^{p, a}= & \frac{f^{p} f}{8} \operatorname{Tr}\left[\lambda_{L R}^{a}\left\{\Sigma^{\dagger} m_{q} \square^{k} \Sigma^{\dagger}+(-1)^{k}\left(\square^{k} \Sigma^{\dagger}\right) m_{q} \Sigma^{\dagger}\right\}\right. \\
& \left.-\lambda_{R L}^{a}\left\{\Sigma m_{q}^{\dagger} \square^{k} \Sigma+(-1)^{k}\left(\square^{k} \Sigma\right) m_{q}^{\dagger} \Sigma\right\}\right] .
\end{aligned}
$$



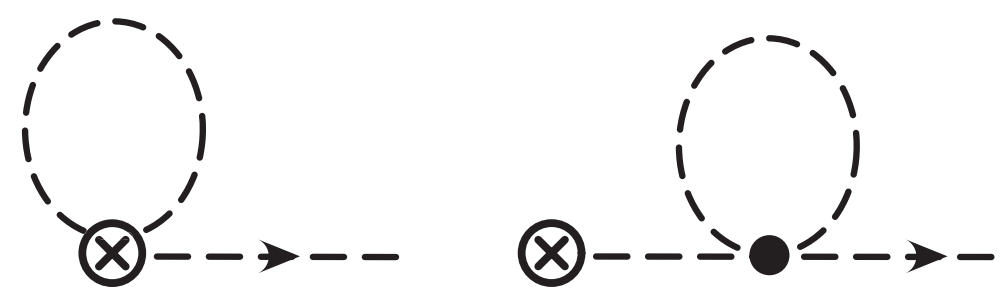

FIG. 1: NLO loop diagrams, where here $\otimes$ denotes an insertion of $\mathcal{O}_{k, 0}^{p, a}$, and the dashed lines are meson fields.

The sum and difference $\overline{\mathcal{O}}_{k, 2}^{p, a} \pm \overline{\mathcal{O}}_{k, 3}^{p, a}$ contain factors of $\left(\Sigma^{\dagger} m_{q} \pm m_{q}^{\dagger} \Sigma\right)$ and $\left(\Sigma m_{q}^{\dagger} \pm m_{q} \Sigma^{\dagger}\right)$. We can trade $\overline{\mathcal{O}}_{k, 2}^{p, a}-\overline{\mathcal{O}}_{k, 3}^{p, a}$ for operators with derivatives on more than one $\Sigma$ by using the equation of motion for $\Sigma$,

$$
\Sigma^{\dagger}\left(i \partial_{\mu}\right)^{2} \Sigma=-\left(i \partial^{\mu} \Sigma^{\dagger}\right)\left(i \partial_{\mu} \Sigma\right)+B_{0}\left(\Sigma^{\dagger} m_{q}-m_{q}^{\dagger} \Sigma\right)
$$

together with the analogous equation for $\Sigma^{\dagger}$. These operators with derivatives on more than one $\Sigma$ do not generate one-meson matrix elements at tree level and can be omitted from our analysis. Thus only $\overline{\mathcal{O}}_{k, 2}^{p, a}+\overline{\mathcal{O}}_{k, 3}^{p, a}$ contributes and for simplicity we trade this for $\overline{\mathcal{O}}_{k, 2}^{p, a}$. We can also insert more $\Sigma$ or $\Sigma^{\dagger}$ fields into $\overline{\mathcal{O}}_{k, 2}^{p, a}$ and get $\operatorname{Tr}\left[\lambda_{L R}^{a} \Sigma^{\dagger} m_{q} \Sigma^{\dagger}\left(\square^{k} \Sigma\right) \Sigma^{\dagger}+\ldots\right]$. But this operator can be reduced to $\overline{\mathcal{O}}_{k, 2}^{p, a}$ using Eq. (25). Finally, we can consider operators where the power suppression is generated by derivatives rather than a factor of $m_{q}$. Boost invariance requires that these operators still have $k$ factors of $n^{\mu}$, so they will involve $\square^{k}$ just like the operators we have been considering. To get power suppression with derivatives we can either use $\left(\partial_{\mu} \Sigma^{\dagger}\right)\left(\partial^{\mu} \Sigma\right)$ which has derivatives on more than one $\Sigma$, or $\Sigma^{\dagger}\left(\partial^{\mu}\right)^{2} \Sigma$ which can be traded for operators with $m_{q}$ 's using Eq. (29). Therefore, the operators with $m_{q}$ 's in Eqs. (26),(27) suffice.

At NLO chiral logarithms can be obtained from loop diagrams involving the LO operators as shown in Fig. 1. For $k=0$ the operator $O_{k=0}^{p, a}$ is the pseudoscalar current whose Fig. 1 graphs give the one-loop corrections to $f_{M}^{p}$. For odd $k$ the one-loop graphs vanish due to the $\left[1+(-1)^{k}\right]$ factor originated from $\mathcal{C}$. For any even $k>0$ the diagrams have a term where all derivatives act on the outgoing meson line, and this gives the same corrections as for $f_{M}^{p}$. The first diagram could have additional contributions from derivatives acting inside the loop but it is straightforward to show that these diagrams vanish identically since $n^{2}=0$, and that the same holds true for LO operators with derivatives on more than one $\Sigma[8]$. Thus, we have shown that all possible non-analytic corrections are contained in $f_{M}^{p}$ at NLO. This is true for every moment, and so we conclude that the leading order SU(3) 
violation of $\phi_{M}^{p}(x)$ is analytic in $m_{q}$.

At NLO the $\overline{\mathcal{O}}_{k, 1}^{p, a}, \overline{\mathcal{O}}_{k, 2}^{p, a}$ and the wave function renormalization counterterms all contribute:

$$
\left\langle M^{b}\left|c_{k, 0} Z_{M}^{1 / 2} \mathcal{O}_{k, 0}^{p, a}+\sum_{i=1}^{2} b_{k, i} \overline{\mathcal{O}}_{k, i}^{p, a}\right| 0\right\rangle=-i f_{M}^{p} \delta^{a b}(n \cdot p)^{k}\left\langle z^{k}\right\rangle_{M}^{p}
$$

For $M=\pi$ and $K, f_{M}^{p}$ can be related to $f_{M}^{P}$ by relating $\bar{\psi} \gamma_{5} \lambda^{a} \psi$ and the derivative of $\bar{\psi} \gamma^{\mu} \gamma_{5} \lambda^{a} \psi$ in the operator level. Acting $D_{\mu}$ on both sides of

$$
\left\langle M^{b}\left|\bar{\psi}(x) \gamma^{\mu} \gamma_{5} \lambda^{a} \psi(x)\right| 0\right\rangle=-i f_{M}^{P} \delta^{a b} p^{\mu} e^{i p \cdot x}
$$

then by the equations of motion and Eq.(12) with $k=0$, one obtains

$$
\begin{aligned}
f_{\pi}^{p} & =f_{\pi}^{P} \frac{m_{\pi}^{2}}{2 \bar{m}}, \\
f_{K}^{p} & =f_{K}^{P} \frac{m_{K}^{2}}{\bar{m}+m_{s}} .
\end{aligned}
$$

These relations should be reproduced to all orders in ChPT. Indeed, direct computations of the leading chiral logarithmic corrections of $f_{M}^{p}, f_{M}^{P}$ and $m_{M}^{2}$ confirm these results. There is no relation between $f_{\eta}^{p}$ and $f_{\eta}^{P}$, however, because

$$
i D_{\mu}\left\langle\eta\left|\bar{u} \gamma^{\mu} \gamma_{5} u+\bar{d} \gamma^{\mu} \gamma_{5} d-2 \bar{s} \gamma^{\mu} \gamma_{5} s\right| 0\right\rangle=-2\left\langle\eta\left|\bar{m}\left(\bar{u} \gamma_{5} u+\bar{d} \gamma_{5} d\right)-2 m_{s} \bar{s} \gamma_{5} s\right| 0\right\rangle
$$

which is not proportional to $\left\langle\eta\left|\bar{u} \gamma_{5} u+\bar{d} \gamma_{5} d-2 \bar{s} \gamma_{5} s\right| 0\right\rangle$ away from the SU(3) limit.

By comparing the $k=0$ and $k \neq 0$ cases in Eq.(30), we obtain

$$
\begin{aligned}
\left\langle z^{k}\right\rangle_{M}^{p}= & \left\langle z^{k}\right\rangle_{0}^{p}+2\left\{\left(1+(-1)^{k}\right) \operatorname{Tr}\left[m_{q}\right] \delta^{a b}\left(b_{k, 1}-b_{0,1} c_{k, 0}\right)\right. \\
& \left.+\operatorname{Tr}\left[m_{q}\left(\lambda^{b} \lambda^{a}+(-1)^{k} \lambda^{a} \lambda^{b}\right)\right]\left(b_{k, 2}-b_{0,2} c_{k, 0}\left(1+(-1)^{k}\right) / 2\right)\right\},
\end{aligned}
$$

where $\left\langle z^{k}\right\rangle_{0}^{p}=\left\langle z^{k}\right\rangle_{M}^{p}$ in the chiral limit and $\left\langle z^{0}\right\rangle_{M}^{p}=1$ is used.

For $k=2 m+1$ (odd moments), the $\left\langle z^{k}\right\rangle_{M}^{p}$ structure yields

$$
\begin{aligned}
\left\langle z^{2 m+1}\right\rangle_{\pi}^{p} & =\left\langle z^{2 m+1}\right\rangle_{\eta}^{p}=0, \\
\left\langle z^{2 m+1}\right\rangle_{K^{+}}^{p} & =\left\langle z^{2 m+1}\right\rangle_{K^{0}}^{p}=\left(m_{s}-\bar{m}\right) b_{2 m+1,2}, \\
\left\langle z^{2 m+1}\right\rangle_{K^{-}}^{p} & =\left\langle z^{2 m+1}\right\rangle_{\bar{K}^{0}}^{p}=-\left(m_{s}-\bar{m}\right) b_{2 m+1,2} .
\end{aligned}
$$

For $k=2 m$ (even moments), the $\left\langle z^{k}\right\rangle_{M}^{p}$ structure yields

$$
\begin{aligned}
\left\langle z^{2 m}\right\rangle_{\pi}^{p} & =\left\langle z^{2 m}\right\rangle_{0}^{p}+2 \bar{m} \alpha_{2 m}^{p}+\left(2 \bar{m}+m_{s}\right) \beta_{2 m}^{p} \\
\left\langle z^{2 m}\right\rangle_{K}^{p} & =\left\langle z^{2 m}\right\rangle_{0}^{p}+\left(\bar{m}+m_{s}\right) \alpha_{2 m}^{p}+\left(2 \bar{m}+m_{s}\right) \beta_{2 m}^{p} \\
\left\langle z^{2 m}\right\rangle_{\eta}^{p} & =\left\langle z^{2 m}\right\rangle_{0}^{p}+\frac{\left(2 \bar{m}+4 m_{s}\right)}{3} \alpha_{2 m}^{p}+\left(2 \bar{m}+m_{s}\right) \beta_{2 m}^{p}
\end{aligned}
$$


where $\alpha_{2 m}^{p}=\left(b_{2 m, 2}-b_{0,2} c_{2 m, 0}\right)$ and $\beta_{2 m}^{p}=2\left(b_{2 m, 1}-b_{0,1} c_{2 m, 0}\right)$. By isospin symmetry and charge conjugation, the even moments of different pion states (or kaon states) are equal. Eq.(36) implies a Gell-Mann-Okubo-like relation:

$$
\left\langle z^{2 m}\right\rangle_{\pi}^{p}+3\left\langle z^{2 m}\right\rangle_{\eta}^{p}=4\left\langle z^{2 m}\right\rangle_{K}^{p}
$$

The moment relations imply the LCDF relations in Eqs.(4),(7). They also imply useful relations among the frequently used Gegenbauer moments, defined by

$$
a_{n}^{M, p}=\frac{2}{2 n+1} \int_{0}^{1} d x C_{n}^{1 / 2}(2 x-1) \phi_{M}^{p}(x),
$$

with $a_{0}^{M, p}=1$. Here $C_{n}^{1 / 2}(z)$ denote the Gegenbauer polynomials which are even (odd) functions of $z$ when $n$ is even (odd).

$$
\phi_{M}^{p}(x)=\sum_{i=0}^{\infty} a_{n}^{M, p} C_{n}^{1 / 2}(2 x-1) .
$$

Eqs.(35)-(37) imply that

$$
\begin{aligned}
4 a_{2 m}^{K, p} & =a_{2 m}^{\pi, p}+3 a_{2 m}^{\eta, p}, \\
a_{2 m+1}^{\pi, p} & =a_{2 m+1}^{\eta, p}=0, \\
a_{2 m+1}^{K^{0}, p} & =a_{2 m+1}^{K^{+}, p}=-a_{2 m+1}^{\overline{K^{0}}, p}=-a_{2 m+1}^{K^{-}, p} .
\end{aligned}
$$

\section{B. The $\phi_{M}^{\sigma}$ Analysis}

Following the analogous procedures, the twist-3 operator $\left(O_{k}^{\sigma, a}\right)^{\mu \nu}$ can be decomposed into

$$
\left(O_{k}^{\sigma, a}\right)^{\mu \nu}=\left(O_{k}^{\sigma, a}\right)_{L R}^{\mu \nu}-\left(O_{k}^{\sigma, a}\right)_{R L}^{\mu \nu}
$$

with

$$
\left(O_{k}^{\sigma, a}\right)_{L R}^{\mu \nu}=\bar{\psi}_{L} \sigma^{\mu \nu} \lambda_{L R}^{a}[i n \cdot \overleftrightarrow{D}]^{k+1} \psi_{R}
$$

and similarly for $\left(O_{k}^{\sigma, a}\right)_{R L}^{\mu \nu}$. Under charge conjugation,

$$
\mathcal{C}^{-1}\left(O_{k}^{\sigma, a}\right)^{\mu \nu} \mathcal{C}=(-1)^{k}\left(O_{k}^{\sigma, a}\right)^{\mu \nu}
$$

if we demand $\lambda_{L R, R L}^{a}$ transform according to Eq. (19). At NLO in the chiral expansion, $O_{k}^{\sigma}$ is matched to LO and NLO hadronic operators $\mathcal{O}_{k}^{\sigma}$ and $\overline{\mathcal{O}}_{k}^{\sigma}$ :

$$
\left(O_{k}^{\sigma, a}\right)^{\mu \nu} \rightarrow \sum_{i} c_{k, i}^{\sigma}\left(\mathcal{O}_{k, i}^{\sigma, a}\right)^{\mu \nu}+\sum_{i} b_{k, i}^{\sigma}\left(\overline{\mathcal{O}}_{k, i}^{\sigma, a}\right)^{\mu \nu}
$$


Again, at LO there is only one hadronic operator

$$
\left(\mathcal{O}_{k, 0}^{\sigma, a}\right)^{\mu \nu}=\frac{f^{\sigma} f}{24}\left[1+(-1)^{k}\right] \operatorname{Tr}\left[\lambda_{L R}^{a}\left(n^{[\nu} \partial^{\mu]} \square^{k} \Sigma^{\dagger}\right)-\lambda_{R L}^{a}\left(n^{[\nu} \partial^{\mu]} \square^{k} \Sigma\right)\right],
$$

where $n^{[\nu} \partial^{\mu]}=\left(n^{\nu} \partial^{\mu}-n^{\mu} \partial^{\nu}\right)$. At NLO, there are two independent operators,

$$
\begin{aligned}
\left(\overline{\mathcal{O}}_{k, 1}^{\sigma, a}\right)^{\mu \nu}= & \frac{f^{\sigma} f}{24}\left[1+(-1)^{k}\right] \operatorname{Tr}\left[m_{q} \Sigma^{\dagger}+\Sigma m_{q}^{\dagger}\right] \operatorname{Tr}\left[\lambda_{L R}^{a} n^{[\nu} \partial^{\mu]} \square^{k} \Sigma^{\dagger}-\lambda_{R L}^{a} n^{[\nu} \partial^{\mu]} \square^{k} \Sigma\right] \\
\left(\overline{\mathcal{O}}_{k, 2}^{\sigma, a}\right)^{\mu \nu}= & \frac{f^{\sigma} f}{24} \operatorname{Tr}\left[\lambda_{L R}^{a}\left\{m_{q}^{\dagger} \Sigma n^{[\nu} \partial^{\mu]} \square^{k} \Sigma^{\dagger}+(-1)^{k}\left(n^{[\nu} \partial^{\mu]} \square^{k} \Sigma^{\dagger}\right) \Sigma m_{q}^{\dagger}\right\}\right. \\
& \left.-\lambda_{R L}^{a}\left\{m_{q} \Sigma^{\dagger} n^{[\nu} \partial^{\mu]} \square^{k} \Sigma+(-1)^{k}\left(n^{[\nu} \partial^{\mu]} \square^{k} \Sigma\right) \Sigma^{\dagger} m_{q}\right\}\right] .
\end{aligned}
$$

These yield the following relations. For $k=2 m+1$ (odd moments),

$$
\begin{aligned}
\left\langle z^{2 m+1}\right\rangle_{\pi}^{\sigma} & =\left\langle z^{2 m+1}\right\rangle_{\eta}^{\sigma}=0, \\
\left\langle z^{2 m+1}\right\rangle_{K^{+}}^{\sigma} & =\left\langle z^{2 m+1}\right\rangle_{K^{0}}^{\sigma}=\left(m_{s}-\bar{m}\right) b_{2 m+1,2}^{\sigma}, \\
\left\langle z^{2 m+1}\right\rangle_{K^{-}}^{\sigma} & =\left\langle z^{2 m+1}\right\rangle_{\bar{K}^{0}}^{\sigma}=-\left(m_{s}-\bar{m}\right) b_{2 m+1,2}^{\sigma} .
\end{aligned}
$$

For $k=2 m$ (even moments),

$$
\begin{aligned}
\left\langle z^{2 m}\right\rangle_{\pi}^{\sigma} & =\left\langle z^{2 m}\right\rangle_{0}^{\sigma}+2 \bar{m} \alpha_{2 m}^{\sigma}+\left(2 \bar{m}+m_{s}\right) \beta_{2 m}^{\sigma}, \\
\left\langle z^{2 m}\right\rangle_{K}^{\sigma} & =\left\langle z^{2 m}\right\rangle_{0}^{\sigma}+\left(\bar{m}+m_{s}\right) \alpha_{2 m}^{\sigma}+\left(2 \bar{m}+m_{s}\right) \beta_{2 m}^{\sigma}, \\
\left\langle z^{2 m}\right\rangle_{\eta}^{\sigma} & =\left\langle z^{2 m}\right\rangle_{0}^{\sigma}+\frac{\left(2 \bar{m}+4 m_{s}\right)}{3} \alpha_{2 m}^{\sigma}+\left(2 \bar{m}+m_{s}\right) \beta_{2 m}^{\sigma},
\end{aligned}
$$

where $\alpha_{2 m}^{\sigma}=\left(b_{2 m, 2}^{\sigma}-b_{0,2}^{\sigma} c_{2 m, 0}^{\sigma}\right)$ and $\beta_{2 m}^{\sigma}=2\left(b_{2 m, 1}^{\sigma}-b_{0,1}^{\sigma} c_{2 m, 0}^{\sigma}\right)$. These imply and

$$
\left\langle z^{2 m}\right\rangle_{\pi}^{\sigma}+3\left\langle z^{2 m}\right\rangle_{\eta}^{\sigma}=4\left\langle z^{2 m}\right\rangle_{K}^{\sigma}
$$

In terms of the Gegenbauer polynomials

$$
\phi_{M}^{\sigma}(x)=6 x(1-x) \sum_{i=0}^{\infty} a_{n}^{M, \sigma} C_{n}^{3 / 2}(2 x-1),
$$

such that the Gegenbauer moments are

$$
a_{n}^{M, \sigma}=\frac{4 n+6}{6+9 n+3 n^{2}} \int_{0}^{1} d x C_{n}^{3 / 2}(2 x-1) \phi_{M}^{\sigma}(x)
$$


with $a_{0}^{M, \sigma}=1$. Here $C_{n}^{3 / 2}(z)$ is an even (odd) function of $z$ when $n$ is even (odd), thus we have

$$
\begin{aligned}
4 a_{2 m}^{K, \sigma} & =a_{2 m}^{\pi, \sigma}+3 a_{2 m}^{\eta, \sigma}, \\
a_{2 m+1}^{\pi, \sigma} & =a_{2 m+1}^{\eta, \sigma}=0, \\
a_{2 m+1}^{K^{0}, \sigma} & =a_{2 m+1}^{K^{+}, \sigma}=-a_{2 m+1}^{\overline{K^{0}}, \sigma}=-a_{2 m+1}^{K^{-}, \sigma} .
\end{aligned}
$$

The normalization $f_{M}^{\sigma}$ is related to $f_{M}^{P}$ for $M=\pi$ and $K$. By contracting

$$
\left\langle M^{b}\left|\bar{\psi}(x) \sigma^{\mu \nu} \gamma_{5} \lambda^{a} i \overleftrightarrow{D^{\alpha}} \psi(x)\right| 0\right\rangle=-\frac{1}{3} f_{M}^{\sigma} \delta^{a b}\left(p^{\mu} g^{\nu \alpha}-p^{\nu} g^{\mu \alpha}\right) e^{i p \cdot x}
$$

with $g^{\nu \alpha}$ and making use of $D^{\mu}\left\langle M^{b}\left|\bar{\psi}(x) \gamma_{5} \lambda^{a} \psi(x)\right| 0\right\rangle=f_{M}^{p} \delta^{a b} m_{M}^{2} p^{\mu} e^{i p \cdot x}$, one obtains

$$
\begin{aligned}
& f_{\pi}^{\sigma}=f_{\pi}^{P}\left(\frac{m_{\pi}^{2}}{2 \bar{m}}\right)\left[1-\left(\frac{2 \bar{m}}{m_{\pi}}\right)^{2}\right], \\
& f_{K}^{\sigma}=f_{K}^{P}\left(\frac{m_{K}^{2}}{\bar{m}+m_{s}}\right)\left[1-\left(\frac{\bar{m}+m_{s}}{m_{K}}\right)^{2}\right] .
\end{aligned}
$$

Direct computations of the chiral logarithms of $f_{\pi}^{\sigma}$ and $f_{K}^{\sigma}$ also confirm these results. Again, $f_{\eta}^{\sigma}$ and $f_{\eta}^{P}$ are not related unless in the $\mathrm{SU}(3)$ limit.

\section{THREE PARTON LCDFS}

The three-parton LCDF $\mathcal{T}_{M}$ for meson $M$ is defined by [34]:

$$
\begin{aligned}
& \langle 0| \bar{q}^{\prime}(y n)[y n, v y n] \sigma_{\mu \nu} \gamma_{5} g G_{\sigma \rho}(\text { vyn }) \lambda^{a}[v y n,-y n] q(-y n)\left|M^{b}(p)\right\rangle \\
= & i f_{M}^{3} \delta^{a b}\left[p_{\sigma} p_{[\mu} g_{\nu] \rho}-(\sigma \leftrightarrow \rho)\right] T_{M}(v, y p \cdot n)
\end{aligned}
$$

where $g$ is the strong coupling constant and $G_{\sigma \rho}$ is the gluon field strength tensor,

$$
T_{M}(v, y p \cdot n)=\int d \underline{\alpha} e^{-i y p \cdot n\left(\alpha^{\prime}-\alpha+v \alpha_{g}\right)} \mathcal{T}_{M}\left(\alpha^{\prime}, \alpha, \alpha_{g}\right),
$$

and

$$
\int d \underline{\alpha}=\int_{0}^{1} d \alpha d \alpha^{\prime} d \alpha_{g} \delta\left(1-\alpha^{\prime}-\alpha-\alpha_{g}\right)
$$


and where $\alpha^{\prime}, \alpha$ and $\alpha_{g}$ are momentum fractions carried by $\bar{q}^{\prime}, q$ and the gluon. For simplicity, we use the gauge $n \cdot A=0$, such that the Wilson lines $[y n, v y n]=[v y n,-y n]=1$. After the operator product expansion, the resulting twist-3 operator is

$$
O_{k, j}^{3, a}=\sum_{i=0}^{k} \bar{q}^{\prime}(i n \cdot \overleftarrow{\partial})^{i} \sigma_{\mu \nu} \gamma_{5} g\left[(i n \cdot \vec{\partial})^{j} G_{\sigma \rho}\right] \lambda^{a}(i n \cdot \vec{\partial})^{k-i} q
$$

where we have suppressed the $\mu, \nu, \sigma$, and $\rho$ indexes on the left hand side. Note that $O_{k, j}^{3, a}$ transforms in the same way as $\left(O_{k}^{\sigma, a}\right)^{\mu \nu}$ under chiral transformation and

$$
\mathcal{C}^{-1} O_{k, j}^{3, a} \mathcal{C}=(-1)^{k} O_{k, j}^{3, a}
$$

Thus the matrix element

$$
\left\langle 0\left|O_{k}^{3, a}\right| M^{b}\right\rangle=i f_{M}^{3} \delta^{a b}\left[p_{\sigma} p_{[\mu} g_{\nu] \rho}-(\sigma \leftrightarrow \rho)\right](n \cdot p)^{k+j} \int d \underline{\alpha}\left(\alpha^{\prime}-\alpha\right)^{k} \alpha_{g}^{j} \mathcal{T}\left(\alpha^{\prime}, \alpha, \alpha_{g}\right)
$$

implies

$$
\begin{gathered}
\mathcal{T}_{\pi(\eta)}\left(\alpha^{\prime}, \alpha, \alpha_{g}\right)=\mathcal{T}_{\pi(\eta)}\left(\alpha, \alpha^{\prime}, \alpha_{g}\right) \\
\mathcal{T}_{K^{+}}\left(\alpha^{\prime}, \alpha, \alpha_{g}\right)-\mathcal{T}_{K^{+}}\left(\alpha, \alpha^{\prime}, \alpha_{g}\right)=-\mathcal{T}_{K^{-}}\left(\alpha^{\prime}, \alpha, \alpha_{g}\right)+\mathcal{T}_{K^{-}}\left(\alpha, \alpha^{\prime}, \alpha_{g}\right) \propto\left(m_{s}-\bar{m}\right), \\
2\left[\mathcal{T}_{K^{+}}\left(\alpha^{\prime}, \alpha, \alpha_{g}\right)+\mathcal{T}_{K^{-}}\left(\alpha^{\prime}, \alpha, \alpha_{g}\right)\right]=\mathcal{T}_{\pi}\left(\alpha^{\prime}, \alpha, \alpha_{g}\right)+3 \mathcal{T}_{\eta}\left(\alpha^{\prime}, \alpha, \alpha_{g}\right)
\end{gathered}
$$

together with $\mathcal{T}_{K^{+}}=\mathcal{T}_{K^{0}}$ and $\mathcal{T}_{K^{-}}=\mathcal{T}_{\bar{K}^{0}}$ by isospin. Eq.(66) was also obtained in Refs. $[33,34]$.

The fact that $O_{0, j}^{3, a},\left(O_{0}^{\sigma, a}\right)^{\mu \nu}$ and $O_{0}^{p, a}$ transform in the same way under chiral transformation implies that the normalization constants $f_{M}^{3}, f_{M}^{\sigma}$ and $f_{M}^{p}$ receive the same leading logarithmic corrections. This is true between $f_{M}^{p}$ and $f_{M}^{\sigma}$ in Eqs. (32) and (59) due to the similar structures of the corresponding LO operators in Eqs. (23) and (47). Also, at NLO the ratio

$$
R_{M}=\frac{f_{M}^{3}}{f_{M}^{\sigma}}
$$

satisfies

$$
R_{K^{+}}-R_{K^{-}} \propto\left(m_{s}-\bar{m}\right)
$$

and

$$
4 R_{K}=R_{\pi}+3 R_{\eta}
$$

Analogous relations also exist for the ratio $f_{M}^{\sigma} / f_{M}^{p}$ at NLO. 
As pointed out in Ref. [34] (see also [30, 33]), the three twist-3 LCDFs are related by the equations of motion. This implies in ChPT, the counterterms associated with the three LCDFs are also related. It can be shown straightforwardly that the $\mathrm{SU}(3)$ relations presented in this work are consistent with the constraints imposed by the equations of motion.

\section{LATTICE QCD CHIRAL EXTRAPOLATIONS}

The first few moments of the twist-2 and twist-3 LCDFs can be calculated using lattice QCD. Currently only the second twist-2 moment of pion $\left\langle z^{2}\right\rangle_{\pi}^{P}$ is computed [24-26]. The latest results on $\left\langle z^{2}\right\rangle_{\pi}^{P}$ have converged to $\left\langle z^{2}\right\rangle^{\overline{M S}}\left(\mu=2.67 \mathrm{GeV}^{2}\right)=0.280(49)_{-0.013}^{+0.030}[25]$ and $\left\langle z^{2}\right\rangle^{\overline{M S}}\left(\mu^{2}=5 \mathrm{GeV}^{2}\right)=0.281(28)[26]$ with the lightest $m_{\pi}$ to be $490 \mathrm{MeV}$ and 550 $\mathrm{MeV}$, respectively. In these two calculations, and many others, chiral extrapolations to the physical point $m_{\pi}=140 \mathrm{MeV}$ were performed. Thus the model-independent results on the $m_{q}$ dependence of LCDFs obtained in Ref. [7] and this work are valuable to reduce the systematic errors in the extrapolations.

A very interesting result we found both in the twist-2 and twist-3 LCDFs is that, in the continuum, all the moments of those distributions are analytic in $m_{q}$ at $\mathcal{O}\left(m_{q}\right)$. Remarkably, this nice feature is retained in a lattice theory with (partially) quenched, finite volume and the leading finite lattice spacing effects included. It is easy to see why. In all the cases, the leading logarithms are generated by one loop diagrams with insertion of the LO $\mathcal{O}_{k, 0}$ operator [with the trace replaced by super-trace when (partially) quenched]. These diagrams correct $c_{k, 0}$ by a universal ( $k$ independent) logarithm which can then be absorbed into the normalization constant. Thus the moments are analytic in $m_{q}$. This feature, however, is only true at $\mathcal{O}\left(m_{q}\right)$. At $\mathcal{O}\left(m_{q}^{2}\right)$, the $m_{q}^{2} \ln ^{2} m_{q}$ corrections to $c_{k, 0}$ are generated by two loop diagrams with insertion of the $\mathrm{LO} \mathcal{O}_{k, 0}$ operator. These corrections are $k$ independent and can be absorbed into the normalization factors. The $m_{q}^{2} \ln m_{q}$ corrections, however, can arise from one-loop diagrams with insertion of the $\mathrm{NLO} \overline{\mathcal{O}}_{k, 1}$ and $\overline{\mathcal{O}}_{k, 2}$ operators which depend on $b_{k, 1}$ and $b_{k, 2}$ and correct $c_{k, 0}$ in a $k$ dependent way, thus the $m_{q}^{2} \ln m_{q}$ corrections cannot be absorbed by the normalization constants. Generalizing the above argument to $\mathcal{O}\left(m_{q}^{i}\right)$, the $m_{q}^{i} \ln ^{i} m_{q}$ corrections can be absorbed by the normalization constants, thus asymptotically, 
the LCDFs has the quark mass dependence ${ }^{1}$

$$
a_{00}+\sum_{i=1}^{\infty} \sum_{j=0}^{i-1} a_{i j} m_{q}^{i} \ln ^{j} m_{q}
$$

where $a_{00}$ and $a_{i j}$ are independent of $m_{q}$.

For different types of lattice simulations, the leading chiral corrections from the one loop diagrams can still be absorbed into the normalization constants, such that the LCDFs remain analytic at NLO. Different types of simulations give different normalization constants and different analytic contributions to the LCDFs. For example, the $\left(2 \bar{m}+m_{s}\right)$ term in Eq.(36) is originated from $\operatorname{Tr}\left[m_{q}\right]$ in Eq.(34). In the (partially) quenched theory, $\operatorname{Tr}\left[m_{q}\right]$ is replaced by $\operatorname{Str}\left[m_{q}\right]$ which is zero in the quenched theory [35] and is $\left(2 \bar{m}_{\text {sea }}+m_{s, \text { sea }}\right)$ in the partially quenched [36] theory, with $m_{q, \text { sea }}$ denoting sea quark masses. It is easy to check that Eq.(37) still holds in spite of (partially) quenching. As for finite volume effects, the counterterm structures are the same as those in the infinite volume calculations since the effects are infrared in origin. Thus in simulations with twisted [37-39] or partially twisted $[40,41]$ boundary conditions for fermions, the leading finite volume effects are absorbed by the normalization constant and the moments are all identical to those in the infinite volume calculations. For lattice fermions whose chiral symmetry is broken by finite lattice spacing $a$, new counterterms in powers of $a$ need to be added to account for the leading finite $a$ effects. Since these counterterms are SU(3) symmetric, Gell-Mann-Okubo-like relations like Eq.(37) will still be true. The same is true for mixed action simulations with chiral valance quarks but Wilson or staggered sea quarks.

\footnotetext{
${ }^{1}$ Consider a generic diagram giving an $\mathcal{O}\left(\epsilon^{d}\right)$ contribution to the twist-2 matrix element $\left\langle M(p)\left|O_{k}^{P}\right| 0\right\rangle$, where $\epsilon \sim p \sim m_{\pi}$. If the diagram has a $\mathcal{O}\left(\epsilon^{k+1+2 n}\right)$ pionic operator insertion, $I$ internal propagators, $L$ loops, and $V_{i}$ vertex of order $\epsilon^{d_{i}}$, then $d=k+1+2 n-2 I+4 L+\sum_{i} V_{i} d_{i}$. After using the topological identity $L=I-\sum_{i} V_{i}$ and removing the $\epsilon^{k+1}$ kinematical factor, this diagram gives a $\mathcal{O}\left(\epsilon^{2 h}\right)$ contribution to the moment $\left\langle z^{k}\right\rangle_{M}^{P}$ with $h=n+L+\sum_{i} V_{i}\left(d_{i} / 2-1\right)$. Since $n \geq 0$ and $d_{i} \geq 2, h \geq L$. In general, the diagram has the contribution $\sum_{i=0}^{L} b_{i} m_{q}^{h} \ln ^{i} m_{q}$, with the power of logarithm not bigger than $L$ and $m_{q} \propto \sqrt{m_{\pi}}=\mathcal{O}(\sqrt{\epsilon})$. The $m_{q}^{h} \ln ^{h} m_{q}$ term can only come from the diagram with an insertion of the LO pionic operator $(n=0)$ and can be absorbed into the normalization. The arguments can be easily generalized to the twist- 3 cases.
} 


\section{COMPARISON WITH LIGHT CONE SUM RULE RESULTS}

The first few Gegenbauer moments for the octet mesons have been computed using light cone sum rules [27-32]. The twist-2 moments $a_{2}^{M, P}$ (defined in the same way as $a_{2}^{M, \sigma}$ in Eq.(54)) were determined in different references. Quite different values for $a_{2}^{\pi, P}(\mu=1$ $\mathrm{GeV}), 0.44$ [27] and 0.20-0.25 [28], are obtained using light cone sum rules. After combining these values with various constraints from experimental data [42, 43], a reasonable range is assigned [43]:

$$
0 \leq a_{2}^{\pi, P}(1 \mathrm{GeV}) \leq 0.3
$$

For moments of $K$ and $\eta$, light cone sum rules of Refs. [32] and [30] yield

$$
a_{2}^{K, P}(1 \mathrm{GeV})=0.16, \quad a_{2}^{\eta, P}(1 \mathrm{GeV})=0.2
$$

respectively. Since the range of $a_{2}^{\pi, P}$ is big, the results in Eqs.(73),(74) are still consistent with the ChPT relation found in Ref. [7];

$$
\rho=\frac{a_{2}^{\pi, P}+3 a_{2}^{\eta, P}}{4 a_{2}^{K, P}}=1+\mathcal{O}\left(m_{q}^{2}\right)
$$

However, this equation could provide a tight constraint among $a_{2}^{M, P}$ since one expects the $\mathcal{O}\left(m_{q}^{2}\right)$ correction to be $10 \%$ at most.

As for $a_{1}^{K^{-}, P}$, recent results yield $a_{1}^{K^{-}, P}=0.05 \pm 0.02$ [44], $0.10 \pm 0.12$ [45] and $0.050 \pm$ 0.025 [46]. The positive sign corresponds to the $s$-quark in a $K^{-}$meson carrying a bigger momentum fraction than the $\bar{u}$-quark.

In Ref. [32], twist-3 Gegenbauer moments are computed (see also [47]):

$$
\begin{aligned}
& a_{2}^{M, p}=30 \eta_{3}^{M}-\frac{5}{2} \rho_{M}^{2} \\
& a_{4}^{M, p}=-3 \eta_{3}^{M} \omega_{3}^{M}-\frac{27}{20} \rho_{M}^{2}-\frac{81}{80} \rho_{M}^{2} a_{2}^{M, P}, \\
& a_{2}^{M, \sigma}=5 \eta_{3}^{M}-\frac{1}{2} \eta_{3}^{M} \omega_{3}^{M}-\frac{7}{20} \rho_{M}^{2}-\frac{3}{5} \rho_{M}^{2} a_{2}^{M, P} .
\end{aligned}
$$

The numerical values of $\eta_{3}^{M}$ and $\omega_{3}^{M}$ are independent of $M$, while $\rho_{M}^{2} \propto m_{M}^{2}=\mathcal{O}\left(m_{q}\right)$ and $a_{2}^{M, P}=a_{2}^{P}\left(1+\mathcal{O}\left(m_{q}\right)\right)$ with $a_{2}^{P}=a_{2}^{M, P}$ in the chiral limit. Eq.(76) satisfies the relations Eqs.(40),(55) and, hence, is consistent with chiral symmetry. 


\section{CONCLUSIONS}

Using chiral symmetry we have investigated the leading $\mathrm{SU}(3)$ violation in the complete set of twist-3 light-cone distribution functions of the pion, kaon, and eta, including the two-parton distributions $\phi_{M}^{p}, \phi_{M}^{\sigma}$ and the three-parton distribution $\phi_{M}^{3}$. It has been shown that terms non-analytic in the quark masses do not affect the shape, and only appear in the normalization constants. Predictive power is retained including the leading analytic $m_{q}$ operators. With the symmetry violating corrections we derive useful model-independent relations between $\phi_{\pi}^{p, \sigma}, \phi_{\eta}^{p, \sigma}, \phi_{K^{+}, K^{0}}^{p, \sigma}$, and $\phi_{\bar{K}^{0}, K^{-}}^{p, \sigma}$. We have also commented on the calculation of the moments of these distributions using lattice method and light-cone sum rules.

We thank Will Detmold, Dan Pirjol and Iain Stewart for useful comments on the manuscript. Support of NSC and NCTS of ROC is gratefully acknowledged.

[1] G.Lepage and S.Brodsky, Phys.Rev.D22, 2157 (1980); A.V. Efremov et al., Phys. Lett. B 94, 245 (1980). V.L. Chernyak and A.R. Zhitnitsky, Phys. Rept. 112, 173 (1984).

[2] M. Beneke, G. Buchalla, M. Neubert, and C.T. Sachrajda, Phys. Rev. Lett. 83, 1914 (1999); Nucl. Phys. B591, 313 (2000);Nucl. Phys. B 606, 245 (2001); M. Beneke and M. Neubert, Nucl. Phys. B 675, 333 (2003).

[3] Y. H. Chen, H. Y. Cheng, B. Tseng and K. C. Yang, Phys. Rev. D 60, 094014 (1999); H. Y. Cheng, C. K. Chua and A. Soni, Phys. Rev. D 71, 014030 (2005).

[4] Y.Y. Keum, H-n. Li and A.I. Sanda, Phys. Lett. B 504, 6 (2001); Phys. Rev. D 63, 054008 (2001); Y. Y. Keum and H. n. Li, Phys. Rev. D 63, 074006 (2001).

[5] C.W. Bauer, S. Fleming and M. Luke, Phys. Rev. D 63, 014006 (2001); C.W. Bauer, S. Fleming, D. Pirjol and I.W. Stewart, Phys. Rev. D 63, 114020 (2001); C.W. Bauer and I.W. Stewart, Phys. Lett. B 516, 134 (2001); C.W. Bauer, D. Pirjol and I.W. Stewart, Phys. Rev. D 65, 054022 (2002);C. W. Bauer, D. Pirjol, I. Z. Rothstein and I. W. Stewart, Phys. Rev. D 70, 054015 (2004); C. W. Bauer, I. Z. Rothstein and I. W. Stewart, arXiv:hep-ph/0510241.

[6] See Table 1 in I.W. Stewart, arXiv:hep-ph/0308185.

[7] J. W. Chen and I. W. Stewart, Phys. Rev. Lett. 92, 202001 (2004).

[8] D. Arndt and M. J. Savage, Nucl. Phys. A697, 429 (2002). 
[9] J. W. Chen and X. Ji, Phys. Lett. B 523, 107 (2001); Phys. Rev. Lett. 87, 152002 (2001).

[10] W. Detmold et al., Phys. Rev. Lett. 87, 172001 (2001); Phys. Rev. D 66, 054501 (2002); ibid 68, 034025 (2003).

[11] J. W. Chen and M. J. Savage, Nucl. Phys. A 707, 452 (2002);Phys. Rev. D 65, 094001 (2002);

J. W. Chen and M. J. Savage, Phys. Rev. D 66, 074509 (2002).

[12] S. R. Beane and M. J. Savage, Nucl. Phys. A 709, 319 (2002).

[13] W. Detmold and C.-J. D. Lin, Phys. Rev. D 71, 054510 (2005).

[14] J. W. Chen and X. Ji, Phys. Rev. Lett. 88, 052003 (2002); A. V. Belitsky and X. Ji, Phys. Lett. B 538, 289 (2002).

[15] N. Kivel and M. V. Polyakov, arXiv:hep-ph/0203264.

[16] M. Diehl, A. Manashov and A. Schafer, Phys. Lett. B 622, 69 (2005).

[17] J. W. Chen and M. J. Savage, Nucl. Phys. A 735, 441 (2004).

[18] N. Kivel, M. V. Polyakov and S. Stratmann, arXiv:nucl-th/0407052; M. C. Birse, J. Phys. G 31, B7 (2005).

[19] S. R. Beane and M. J. Savage, Nucl. Phys. A761, 259 (2005).

[20] B. C. Tiburzi, arXiv:hep-ph/0508112.

[21] B. Grinstein and D. Pirjol, Phys. Lett. B 615, 213 (2005); V. Cirigliano and D. Pirjol, arXiv:hep-ph/0508095.

[22] J. W. Chen and W. Detmold, Phys. Lett. B 625, 165 (2005).

[23] T. Huang, X. H. Wu and M. Z. Zhou, Phys. Rev. D 70, 014013 (2004).

[24] A. S. Kronfeld and D. M. Photiadis, Phys. Rev. D 31, 2939 (1985); G. Martinelli and C. T. Sachrajda, Phys. Lett. B 190, 151 (1987); D. Daniel, R. Gupta and D. G. Richards, Phys. Rev. D 43, 3715 (1991); L. Del Debbio, M. Di Pierro, A. Dougall and C. T. Sachrajda [UKQCD collaboration], Nucl. Phys. Proc. Suppl. 83, 235 (2000).

[25] L. Del Debbio, M. Di Pierro and A. Dougall, Nucl. Phys. Proc. Suppl. 119, 416 (2003).

[26] M. Gockeler et al., [QCDSF/UKQCD collaboration] arXiv:hep-lat/0510089.

[27] V. M. Braun and I. E. Filyanov, Z. Phys. C 44, 157 (1989) [Sov. J. Nucl. Phys. 50, 511 (1989); Yad. Fiz. 50, 818 (1989)].

[28] S. V. Mikhailov and A. V. Radyushkin, Phys. Rev. D 45, 1754 (1992).

[29] T. Huang, B. Q. Ma and Q. X. Shen, Phys. Rev. D 49, 1490 (1994).

[30] P. Ball, JHEP 9901, 010 (1999). 
[31] P. Ball, JHEP 9809, 005 (1998).

[32] P. Ball and M. Boglione, Phys. Rev. D 68, 094006 (2003).

[33] A. Hardmeier, E. Lunghi, D. Pirjol and D. Wyler, Nucl. Phys. B 682, 150 (2004).

[34] V. M. Braun and I. E. Filyanov, Z. Phys. C 48, 239 (1990) [Sov. J. Nucl. Phys. 52, 126 (1990)]; V. M. Braun, G. P. Korchemsky and D. Müller, Prog. Part. Nucl. Phys. 51, 311 (2003).

[35] C. W. Bernard and M. F. L. Golterman, Phys. Rev. D 46, 853 (1992); S. R. Sharpe, Phys. Rev. D 46, 3146 (1992); J. N. Labrenz and S. R. Sharpe, Phys. Rev. D 54, 4595 (1996).

[36] C. W. Bernard and M. F. L. Golterman, Phys. Rev. D 49, 486 (1994); S. R. Sharpe, Phys. Rev. D 56, 7052 (1997) [Erratum-ibid. D 62, 099901 (2000)]; S. R. Sharpe and N. Shoresh, Phys. Rev. D 64, 114510 (2001).

[37] M. Luscher, S. Sint, R. Sommer and P. Weisz, Nucl. Phys. B 478, 365 (1996).

[38] P. F. Bedaque, Phys. Lett. B 593, 82 (2004).

[39] G. M. de Divitiis, R. Petronzio and N. Tantalo, Phys. Lett. B 595, 408 (2004).

[40] C. T. Sachrajda and G. Villadoro, Phys. Lett. B 609, 73 (2005).

[41] P. F. Bedaque and J. W. Chen, Phys. Lett. B 616, 208 (2005).

[42] A. Schmedding and O. I. Yakovlev, Phys. Rev. D 62, 116002 (2000); M. Diehl, P. Kroll and C. Vogt, Eur. Phys. J. C 22, 439 (2001); A. P. Bakulev, S. V. Mikhailov and N. G. Stefanis, Phys. Lett. B 508, 279 (2001) [Erratum-ibid. B 590, 309 (2004)]; Phys. Rev. D 67, 074012 (2003); A. P. Bakulev, K. Passek-Kumericki, W. Schroers and N. G. Stefanis, Phys. Rev. D 70, 033014 (2004) [Erratum-ibid. D 70, 079906 (2004)].

[43] P. Ball and R. Zwicky, Phys. Lett. B 625, 225 (2005).

[44] A. Khodjamirian, T. Mannel and M. Melcher, Phys. Rev. D 70, 094002 (2004).

[45] V. M. Braun and A. Lenz, Phys. Rev. D 70, 074020 (2004).

[46] P. Ball and R. Zwicky, arXiv:hep-ph/0510338.

[47] T. Huang, X. H. Wu and M. Z. Zhou, Phys. Rev. D 70, 014013 (2004); T. Huang, M. Z. Zhou and X. H. Wu, Eur. Phys. J. C 42, 271 (2005). 